Health and Medicine | Dr Catherine Aldred \& Professor Jonathan Green

\title{
PACT:
}

Working with parents and carers to help autism development affecting over $1 \%$ of people its symptoms. PACT is an intervention working through in UK national guidance

Autism is an enduring condition evidenced therapy to reduce early social communication parents and carers that is the term improvemontrited longsymptoms after therapy within a clinical trial. This relatively low intensity approach is referenced for autism treatment and is being disseminated through professional training in the UK and worldwide through the MPACT training programmme.

utism is a common
neurodevelopmental disorder
affecting over $1 \%$ of children ind young people globally. It arises early in development with often profound effects on social understanding and communication and patterns of thinking and behaviour; effects that usually endure through the lifespan. Additionally, a quarter of people with no orveny little languag. All this inevitably affects social skills, education, confidence, and ability to find a job. Not only is this stressful for the individual and family, but providing treatment and support for people with autism for their whole life is expensive. Estimated lifetime costs, including medical costs, education, social care, and productivity losses, are estimated at around f1-1.5 million per individual in the UK and between $\$ 1.4$ to $\$ 2.4$ million in the USA. Ideally, children diagnosed at an early age would benefit from immediate and effective support to develop their social communication and development. The is some evidence to show short-term including parent-child engagement, symbolic play and social imitation. However, effective early treatments tha alter the long-term course of the disord have been more difficult to demonstrate.

Dr Catherine Aldred, a consultant

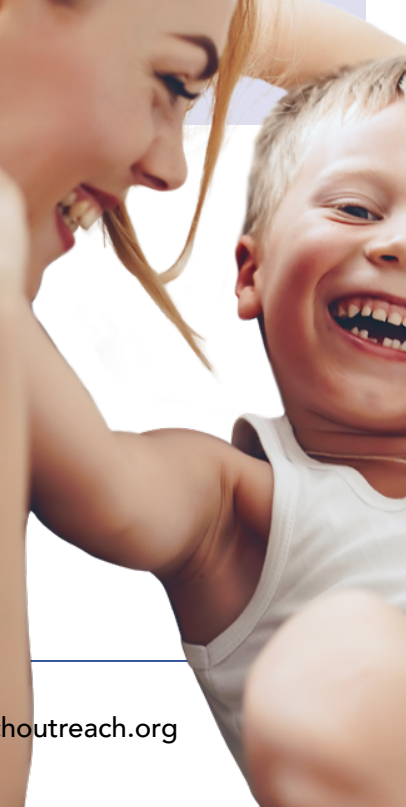

Newcastle, have over 15 years developed new approach, using videofeedback techniques with parents to help them understand and respond to the particular communication style of their young child with autism. The Paediatric Autism Communication Therapy (PACT) aims to help autistic children to develop their early social communication skils. A not-for-profit community interest for Paediatric Autism Communication Therapy (IMPACT) has been set up to deliver PACT training to professionals so as to help extend implementation of evidence-based interventions for children with autism in the UK and internationally.

\section{WHAT IS PACT?}

improve communication and interaction skills in children on the autism spectrum along with patterns of restrictive and repetitive behaviour. An important feature of this method is the way it links the familiarity and skills of adults who know the child best - their parents or carers - with the specialist of therapists.

The process involves recording sessions between parents or carers playing or watch the videos and, with the help of the therapist, learn to recognise subtle moments of interaction with their child. The idea is to help parents to be able to create opportunities for communicatio and adapted interaction in their dayto-day lives. As one parent reflects '.. the video was like looking through a magnifier, I could see so much more, now I feel I know him better, "m more skilled in interacting and communicating with him. You have changed our lives.'

A key feature of PACT is the way it uses video-feedback to work through parents

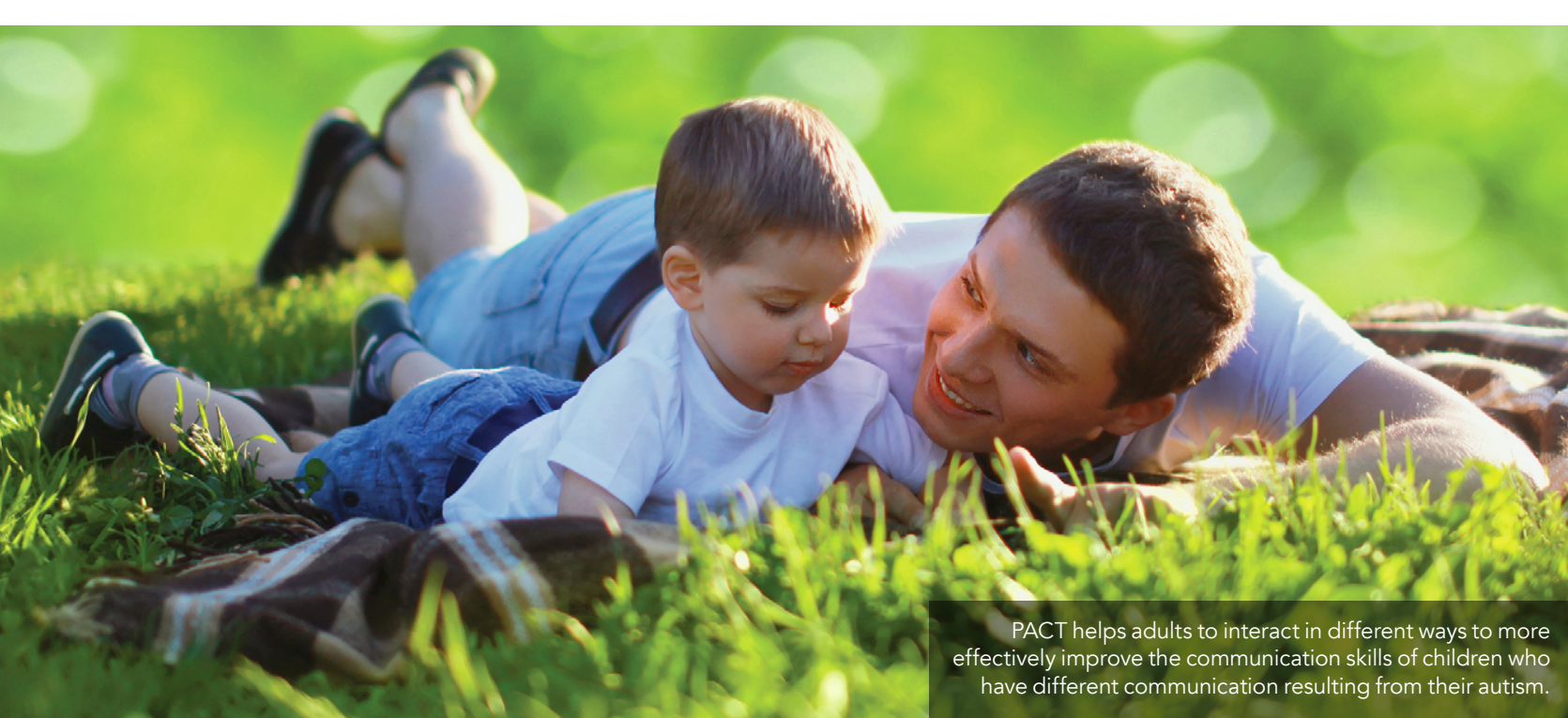
family life.

PACT aims to help autistic children to develop their early social communication skills. able to encourage and empower parents and carers, allowing them to develop key skills shown to improve the child's social communication outcomes. Professionals are trained to identify both the child's and the parent's existing abilities and build on them to extend communication skills to the highest possible level.

In practice, the therapy consists of twelve 1.5 hour fortnightly sessions over six months, with optional monthly maintenance sessions over a further six months. In comparison with other reatments that may require up to 30 relatively low intensity and may be feasible for working parents. Between sessions, parents are asked to practice PACT strategies for at least 30 minutes daily during play or natural interactions with their child. In this way, PACT is gradually introduced into everyday

EVIDENCE-BASED APPROACH PACT is the only intervention of this type to date to have shown sustained symptom improvement in children with autism over the long-term on rigorous testing. A multi-centre randomised controlled trial involved 152 autistic children in Manchester, Newcastle and London, aged two to four years old, half in addition to usual care, and half usual care only (Green et al., Lancet, 2010). Taken as a group, children who received PACT showed greater improvement in social communication and repetitive restricted behaviour symptoms whe care alone. Significant gains in parentchild interaction (shown in other analysis to mediate the child symptom change) were seen at six months. Researcher-rated language skills was an area that did not improve. Parents reported fewer difficulties in all the core
symptoms associated with autism: social interaction, social communication, interests in the PACT group, compared

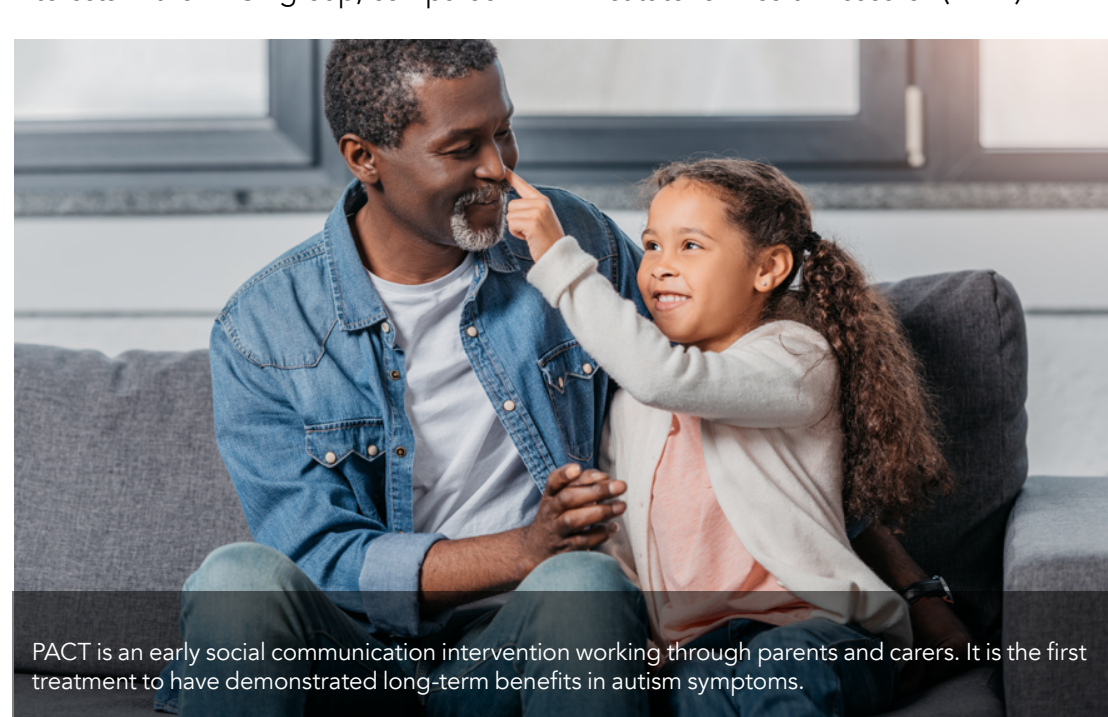
end of the original intervention (Pickles et al., Lancet, 2016). $80 \%$ of the original 152 trial participants were assessed in middle childhood by researchers who did not know if a child had had the PACT autistic, but the relative improvement autism symptom severity, child social initiations, and reduced restricted

epetitive and stereotyped behaviours wa sustained. This is a striking result, since in atism often the effects of intenention a found to 'wash out' over time.

This trial was selected by the UK National to usual care, as well as improved

Importantly, these relative improvements were still apparent when the children 


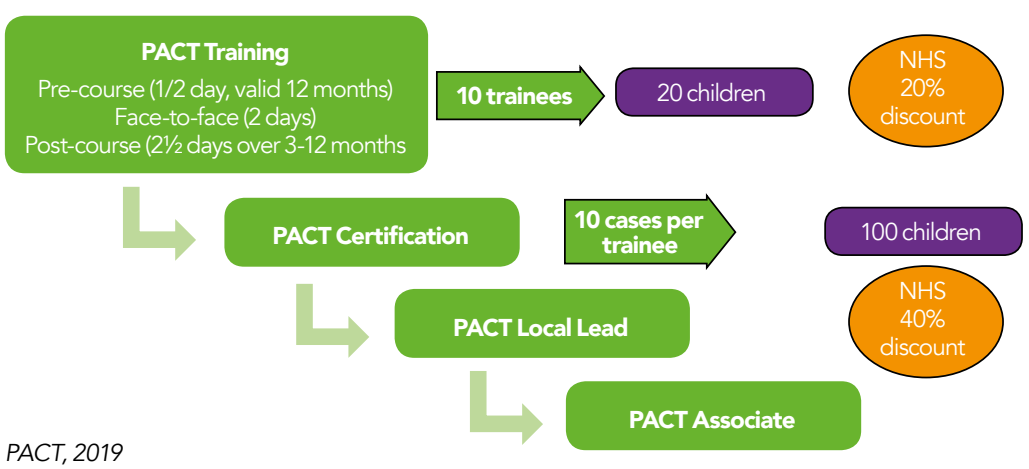

Pre-school PACT has been rigorously tested as to its sustained benefits on autism symptoms into middle childhood.

as a 'Signal Study' for its "high quality "makers" (doi: 103310/signel000381). PACT was recently added to the ecommended curriculum for the Childer and Young People's Improving Access to Psychological Treatment (IAPT) programme, overseen by NHS England and Health Education England. Full information about the research behind PACT can be found at: $h$ tttp://research. bmh.manchester.ac.uk/pact/about.

\section{RAINING IN PACT}

To address the treatment gap for people with autism, the goal of IMPACT is to

maximise the reach of PACT intervention PACT leads in their locality This is dome PACT leads in their localty. This is done PACT post-course pratice worport ocalities, and the global'Train the' Trainer' programme. This programme allows individual international centres to become centres of excellence by developing PACT training in their own communities with support from IMPACT. The PACT local leads programme has been successfully implemented in reas in the UK and 'Train the Trainer' programme is being implemented internationally. Thus far, 240 professionals have trained or booked to be trained during 2017-19; originating from UK, France, Spain, laly, Germany, Switzerland, Hong Kong, Turkey, USA, Australia, Canada, and Argentina. The qually of the PACT senices delivered wilbe moniter through local

and feedback from parents, carers and professionals.

PACT training is offered for healthcare and other professionals who have experience of working with families of children with autism. To become a PACT practitione training starts with a half day digital e-learning as a way of introduction to professionals to gain general awareness of PACT but does not qualify the practition to deliver the intervention in practice. Registration for e-learning introduction can be done online via Hogrefe publish at https.//uww.hogrefe.co.uk/e-learning/ pact-e-learning-information.

Subsequent to this, there is a clinical PACT training for professionals who wish Chain of effect Parent/carer
interaction changes leadsto Change in child's
interaction with (a) Improved child
interaction with interaction with
others - sympton
reduction PACT, 2017

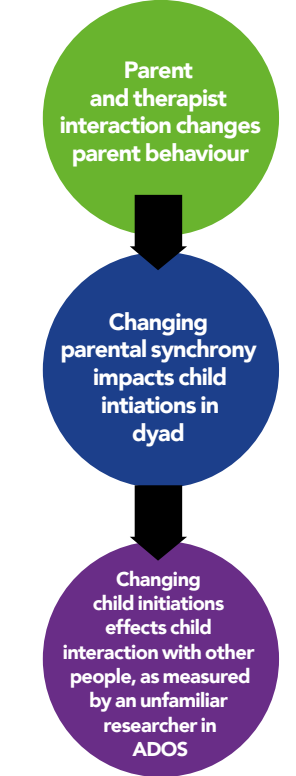

clinical setting or in research studies Registration for this stage can be done co.uk): it involves attending a two-day group training session followed by practice supenvision in which trainees submit two case studies for assessment including the videos of the adult-child play and feedback to the parents/carers.

Later, to be able to teach the method to others, participants may progress to become a UK PACT lead or international trainer. Progress to this can come from successfully completing at least five clinical cases, plus co-training a PACT two-day live group course and co-rating tho post-course videos. To find out more 政 training, please visit https:// pacttraining.co.ukl.

\section{GLOBAL REACH}

The evidence for PACT is named in

UK and Netherlands PACT research studies in other countries are ongoing PACT is being trained and implemented in more than 12 countries worldwide; reaching communities through training hubs in Italy, France, Spain, and Hong Kong. The PACT approach has been adapted with success to be used in low resource settings; international colleagues have partnered with Professor Green's group to adapt the PACT intervention for use in Low and Medium Income (LMIC) settings (http://research.bmh. manchester.ac.uk/pact/PASS/. In 2016 thial of the adapted manual was completed involving 65 children with good feasibility and acceptability and that as a group, the participating children improved their communication skills in a similar manner to the results obtained in the UK trial (Rahman et al, 2016). This project demonstrated for the first time that an autism treatment developed in a high-income country UK setting could be dapted for LMIC countries, emphasising the feasibility and potential of this method for adaptability into different global communities.

The hope is that, through widespread training and further testing of its application and user feedback in be made act and cultures across the world.

\section{Behind the Research}

Catherine Aldred

\section{$\approx=$ \\ Jonathan Green}

E: info@pacttraining.co.uk T: 07967148321 W: www.pacttraining.co.uk; http://research.bmh.manchester.ac.uk/pact/about/ W: https://www.hogrefe.co.uk/e-learning/pact-e-learning-information

\section{Research Objectives}

Professional training and service implementation of PACT therapy, to help address the gap in evidenced-based early intervention for children with autism, both in the UK and internationally.

\section{Detail}

Bio

Directors of IMPACT are Dr Catherine Aldred, a Speech and Language Therapist who initiated the PACT approach, and Rrofessor Jonathan Green, a Child Psychiatist, based at the University of Manchesterwho led the research programm teams and collaborating institutions can be found a thtp research.bmh.manchester.ac.uk/pact/about.

Funding

Funding for PACT trials came from: i) Shirley Foundation, for the pilot study, ili) UKMedical Research Council and Department for Educationl for PACT and PACT 711 studies, iii) MRC/National Institute for Health Research Efficacy and Mechanism programme, for the current PACT-G study. iv) Autism Speaks, Grand Challenges Canada, MRC/ Welcome/UK DFID Joint Global Health Trials, for the desting in South Asia.

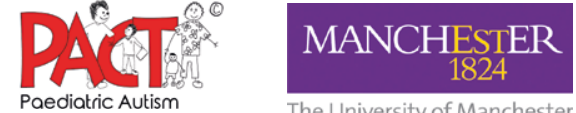

Collaborating Teams

A majority of the evidence for PACT comes from a Professor Jonathan Green which insurtes the fol by principle investigators and institutions:

University of Manchester and Royal Manchester Children's Hospital: Dr Catherine Aldred - Dr Kathy Leadbitter Newcastle University. - Prof Ann Le Couteur - Prof Helen McConachi - DrVicki Grahame

\section{References}

Aldred C, Green J, Adams C. (2004). A new social communication intervention for children with autism: pilot randomised controlled treatment study suggesting
effectiveness. J Child Psychol Psychiatry. 45(8):1420-30. https://doi.org/10.1111/j.1469-7610.2004.00338. Green J, Charman T, McConachie H, Aldred C, Slonims V, Howlin, et al. (2010). Parent-mediated communicationrandomised controlled trial. The Lancet. 375(9732):2152-2160. https://doi.org/10.1016/50140-6736(10)60587-9.

Pickles A, et al. (2016). Parent-mediated social communication therapy for young children with autism (PACT): long-term
follow-up of a randomised controlled trial. The Lancet. 388(10059), 2501-9. DOI: https://doi.org/10.1016/S0140$6736(16) 31229-6$

Rahman A, Divan G, Hamdani SU, Vajaratkar V, Taylor C,
Leadbitter K, et al. (2016). Effectiveness of the parentmediated intervention for children with autism spectrum disorder in south Asia in India and Pakistan (PASS): a 136. D-15-00305R DOl. 10.1016/S2215-0366(15)00388Website: https://pacttraining.co.uk/

\section{Personal Response}

PACT has been well received by parents, care children and healthcare providers. How long do you

II We're delivering PACT training in NHS local teams in different parts of the UK in 2019 with the aim of gaining implementation. We're helping NHS services connect to share and extend successful PACT implementation in the

UK. We hope to be able to extend PACT training to most
NHS services over the next five years.

urther Information

Building on the evidence generated during the PACT consortium and global health programme, IMPACT (Interaction Method for Paedic community interest company set up to promote the training 